\title{
Evolutionary engineering of Wickerhamomyces subpelliculosus and Kazachstania gamospora for baking
}

Thandiwe Semumu ( $\nabla$ thandiwesemumu@gmail.com )

Botswana International University of Science and Technology https://orcid.org/0000-0002-0451-4856

\section{Amparo Gamero}

University of Valencia: Universitat de Valencia

\section{Teun Boekhout}

Westerdijk instutute

Nerve Zhou

Botswana international university of Science and Technology

\section{Research Article}

Keywords: alternative baker's yeasts, non-conventional yeasts, evolutionary engineering

Posted Date: November 3rd, 2021

DOI: https://doi.org/10.21203/rs.3.rs-1020169/v1

License: (c) (1) This work is licensed under a Creative Commons Attribution 4.0 International License.

Read Full License 


\section{Evolutionary engineering of Wickerhamomyces subpelliculosus and Kazachstania gamospora for baking}

$1 \quad *$ Thandiwe Semumu $^{1}$, Amparo Gamero ${ }^{2}$, Teun Boekhout ${ }^{3,4}$ and ${ }^{*}$ Nerve Zhou $^{1}$

$2{ }^{1}$ Department of Biological Sciences and Biotechnology, Botswana International University of

3 Science and Technology, Private Bag 16, Central District, Palapye, Botswana

$4 \quad{ }^{2}$ Department of Preventive Medicine and Public Health, Food Science,Toxicology and Forensic

5 Medicine, Faculty of Pharmacy, University of Valencia, Avda. Vicent Andrés Estellés S/N, 46100

6 Burjassot (València), Spain

$7 \quad{ }^{3}$ Westerdijk Fungal Biodiversity Institute, Utrecht, The Netherlands

$8{ }^{4}$ Institute of Biodiversity and Ecosystem Dynamics (IBED), University of Amsterdam, Amsterdam,

9 The Netherlands

10 *Corresponding Authors

11 thandiwe.semumu@studentmail.biust.ac.bw and zhoun@biust.ac.bw

13 Keywords: alternative baker's yeasts, non-conventional yeasts, evolutionary engineering

\section{Abstract}

15 The conventional baker's yeast, Saccharomyces cerevisiae, is an indispensable baking workhorse of 16 all times. Its monopoly coupled to its major drawbacks such as streamlined carbon substrate 17 utilisation base and a poor ability to withstand a number of baking associated stresses prompt the 18 need to search for alternative yeasts to leaven bread in the era of increasingly complex consumer

19 lifestyles. Our previous work identified the inefficient baking attributes of Wickerhamomyces 20 subpelliculosus and Kazachstania gamospora as well as preliminarily observations of improving 21 fermentative capacity of potential alternative baker's yeasts using evolutionary engineering. Here we 22 report the characterisation and improvement in baking traits in five out of six independently evolved 23 lines incubated for longer time and passaged for at least 60 cycles relative to their parental strains as 
well as the conventional baker's yeast. In addition, evolved clones produced bread with a higher loaf volume when compared to bread baked with either ancestral strain or the control conventional baker's yeast. Remarkably, our approach improved the yeasts' ability to withstand baking associated stresses, a key baking trait exhibited poorly in both the conventional baker's yeast and their ancestral strains. W. subpelliculosus evolved the best characteristics attractive for alternative baker's yeasts as compared to the evolved $K$. gamospora strains. These results demonstrate the robustness of evolutionary engineering in development of alternative baker's yeasts.

\section{Introduction}

33 The conventional baker's yeast, Saccharomyces cerevisiae, remains the baking workhorse of all 34 times. Baker's yeast is a key ingredient in baking that serves three functions; production of $\mathrm{CO}_{2}$, 35 dough maturation, and development of flavour of bread and other farinaceous products (Attfield, 36 1997, Kariluoto et al., 2004). In addition, to its baking attributes, S. cerevisiae was the first yeast to 37 be domesticated (Fay \& Benavides, 2005), the first eukaryote to be completely sequenced (Goffeau et al., 1996), the first model organism (Botstein et al., 1997), the first genetically modified food producing organism (Aldhous, 1990), and it is the most studied yeast (Liti et al., 2009). This list shows why baker's yeast is the yeast of choice and probably the main reason behind its millennialong monopoly of not only the baking industry, but also the wine and beer industries (Gibson et al., 2017). The primary role of a baker's yeast lies in its ability to rapidly ferment sugars (mainly maltose) found in flour production (Randez-Gil et al., 1999) and subsequent accumulation of $\mathrm{CO}_{2}$. Yeast determines the overall quality of the bread beyond just gas production, such as bread aroma, texture, crumb structure and overall appearance of the bread. However, the conventional baker's yeast has challenges impacting the overall bread quality, which therefore affects its continued use in modern consumer-driven markets.

Firstly, Saccharomyces cerevisiae has a stream-lined utilisation of carbon sources (Ostergaard et al., 2000), produces a low diversity of secondary metabolites, and has poor ability to resist baking associated stresses as compared to recently described nonconventional yeasts (Hernández-López \&

51 Vargas-Albores, 2003, Struyf et al., 2017). Incomplete sugar fermentation leads to an unhealthy diet

52 in our modern lifestyles where reduction of intake of sugars remains the cornerstone to fight obesity 53 and diabetes. The production of less diverse secondary metabolites leads to poor development of 
54 bread flavour (Harvey et al., 2010, Aslankoohi et al., 2016). During baking, there are baking55 associated stresses that yeast may encounter such as high osmotic stress, high oxidative stress, 56 ethanol stress and others as well as low/high temperatures during baking and storage (Attfield, 1999).

57 However, the conventional baker's yeast has a poor ability to withstand stressful conditions, which 58 ultimately leads to reduced product yields. Conditions, such as high osmotic pressure associated with 59 downstream processing during starter culture production as well as during fermentation of dough 60 with high amounts of sugars and salts and low $\mathrm{pH}$ in some dough, have been well documented 61 (Attfield, 1997, Hernández-López \& Vargas-Albores, 2003, Takagi, 2017). Fermentation generates 62 reactive oxygen species, which exert an oxidative stress to yeast (Takagi, 2017) and the conventional 63 baker's yeasts is poor in responding to oxidative stress (Takagi, 2017). Ethanol formed during 64 fermentation, exerts major stress to yeast cells, and acts as a membrane defacer, which the 65 conventional baker's yeast can withstand. These many challenges create a need to find alternative yeasts presenting robust baking traits.

67 The ability to ferment sugars and produce $\mathrm{CO}_{2}$ is not circumscribed to $S$. cerevisiae (Hagman et al., 2013). Recent advances on these non-conventional yeasts as producers of desirable and unique aromas (Gamero Lluna \& de Jong, 2013, Gamero et al., 2016, Ravasio et al., 2018), their resistance to many industry associated stresses (Radecka et al., 2015) suggest their applicability in baking. We previously the screening of two potential non-conventional yeasts, $W$. subpelliculosus and $K$. gamospora, as alternative baking yeasts (Zhou et al., 2017). These yeasts exhibited a poor fermentative capacity and poor dough leavening attributes when compared to the conventional baker's yeast. Preliminary dough leavening abilities after adaptively evolving these yeasts in flour dough highlighted serial passing in flour dough could be used to improve baking traits (Zhou et al., 2017). In this study we set a longer incubation time ( $48 \mathrm{~h}$ ) coupled to a relatively higher number of passaging cycles (at least 60) as compared to the parameters reported in (Zhou et al., 2017) and showed that evolutionary engineering is an attractive strain improvement strategy to increase the gassing power, dough leavening and baking associated stress tolerance of alternative baker's yeasts. In brief, we report the improvement in the baking attributes of clones from 5 out of 6 of independently and parallel-evolved lines when compared to their respective ancestral strains as well as the control conventional baker's yeast. In addition, this work further reports a similar trend in

83 improvements of baking traits based on baking trials, thus confirming our hypothesis that 84 evolutionary engineering improves baking traits. 


\subsection{Strains}

87 The two potential alternative baker's yeasts, W. subpelliculosus (CBS 5552) and K. gamospora (CBS

88 10400) reported in our previous work (Zhou et al., 2017) were used for evolutionary engineering 89 experiments. A control conventional baker's yeast from Anchor Yeast as instant dry yeast $(S$. 90 cerevisiae) sold in retail outlets was selected as a positive control.

\subsection{Culture media}

92 Yeast Peptone Maltose (YPM) media constituted of $2 \%$ Maltose, $0.5 \%$ Yeast extract and $1 \%$

93 Peptone), at a $\mathrm{pH}$ of 6.2 was used to revive isolates stored at $-80{ }^{\circ} \mathrm{C}$. Yeast peptone dextrose (YPD)

94 media constituting of $2 \%$ Glucose, $0.5 \%$ Yeast extract and $1 \%$ Peptone) adjusted to a pH of 6.2

95 using $1 \mathrm{M} \mathrm{NaOH}$ and $1 \mathrm{M} \mathrm{HCl}$ was used for stress tolerance tests.

\subsection{Evolutionary engineering scheme}

97 Evolution experiments were conducted by serially passaging yeast in wheat flour dough as reported 98 previously (Zhou et al., 2017) with modifications of flour dough preparations (shorter sterilisation 99 time and lower sterilisation temperature), longer duration of incubation times and an increased 100 number of passaging cycles. In brief, flour dough was prepared by mixing $0.6 \mathrm{~g}$ of wheat flour 101 (sterilised by heating for $48 \mathrm{~h}$ at $70{ }^{\circ} \mathrm{C}$ ) and sterile $500 \mu \mathrm{L}$ of $0.5 \mathrm{M} \mathrm{NaCl}$ in $2 \mathrm{~mL}$ Eppendorf microtubes. An isogenic colony from each of the master plates (W. subpelliculosus (CBS 5552) and K. gamospora (CBS 10400)) was grown overnight in YPM media and harvested thereafter and set to an $\mathrm{OD}_{600 \mathrm{~nm}}$ of 1. Triplicates (parallel lineages) from each isogenic colony were then inoculated into wheat flour dough and microtubes were then incubated without shaking at $30{ }^{\circ} \mathrm{C}$ (see Supplementary Materials, Figure S1). Parallel lines from $K$. gamospora were designated as $K g_{-} 1, K g \_2$, and $K g \_3$,

107 whereas those from W. subpelliculosus were designated as Ws_1,Ws_2, and Ws_3. After 48 h of 108 incubation, a toothpick-full of dough was transferred into fresh dough to start another passage. This 109 procedure was repeated for several passages (see Supplementary Materials, Figure S2) until the 110 microtubes popped open before the set $48 \mathrm{~h}$ incubation time. After every passage, the tubes were 111 cryopreserved at $-80{ }^{\circ} \mathrm{C}$ in $25 \%$ glycerol for further studies and as starter culture in case of a mishap 112 during evolution such as contamination. After passaging for 60 passages cells from a toothpick from 113 each of the parallel-evolved lines were serially dilution plated out and used for characterisation 
114 experiments. To be specific, we selected the biggest colony from each of the 6 parallel-evolved lines

$115\left(K g \_1, K g \_2\right.$ and $K g \_3$ from $K$. gamospora evolved lines and $W s_{-} 1, W s \_2$ and $W s \_3$ from $W$.

116 subpelliculosus evolved lines) and characterised the change in baking attributes when compared to

117 their ancestral strains as well as those of the conventional baker's yeast.

\subsection{Confirmation of evolving strains and detection of contamination}

120 After every 10 passages, the absence of contamination was verified by microscopy and sequencing.

121 For sequencing, DNA extraction was carried out using ZR Soil Microbe DNA kit ${ }^{\mathrm{TM}}$ (Zymo Research,

122 Orange, CA, USA) according to manufacturers' recommendations. A 560 - 750 bp amplicon size

123 was amplified from ITS-5.8S rDNA using ITS1 (5'-TCCGTAGGTGAACCTGCGG-3') and ITS4

124 (5'-TCCTCCGCTTATTGATATGC-3'). The following PCR program: Initial denaturation: $94{ }^{\circ} \mathrm{C}-4$

125 min, cycle denaturation: $94{ }^{\circ} \mathrm{C}-30 \mathrm{sec}$, primer annealing: $54{ }^{\circ} \mathrm{C}-30 \mathrm{sec}$, chain extension: $72{ }^{\circ} \mathrm{C}-$

$1261 \mathrm{~min}$, chain elongation: $72{ }^{\circ} \mathrm{C}-7 \mathrm{~min}$, number of cycles: 36 cycles, and holding temperature: $4^{\circ} \mathrm{C}$

127 was run. PCR products were analysed by gel electrophoresis (using $1 \%$ agarose gel in 1X TBE

128 buffer) and then purified before being quantified using an ND- 1000 spectrophotometer (NanoDrop;

129 Thermo Scientific, Wilmington, DE, USA). Inqaba Biotechnical Industries in Pretoria, South Africa

130 sequenced the purified PCR products. SnapGene sequence editing tool was used for removing 131 ambiguous bases (http://www.snapgene.com). The yeasts were identified by searching databases 132 using BLAST sequence analysis tool (http://www.ncbi.nlm.nih.gov/BLAST/). To confirm the 133 identity, pairwise identification database owned by the Westerdijk Fungal Biodiversity Institute 134 (CBS-NAW) (http://www.westerdijkinstitute.nl/) was also used.

\subsection{Investigation of fermentative capacity}

137 To investigate the improvement in fermentative capability of the evolved strains, fermentation was 138 carried out using YPM in $60 \mathrm{~mL}$ BD Luer-Lok ${ }^{\mathrm{TM}}$ syringes (BD® Syringes) as described in our 139 previous work (Zhou et al., 2017). A single colony (selected based on colony size) from each of the 140 evolved lineages of $W$. subpelliculosus (Ws_1,Ws_2, and $W s_{-} 3$ ) was grown overnight in $2 \mathrm{~mL}$ of 5 $141 \%$ YPM in $5 \mathrm{~mL}$ culture tubes at $26^{\circ} \mathrm{C}$ at $200 \mathrm{rpm}$ on a shaker (Infors HT). The yeasts were then 142 harvested, washed and used to inoculate $5 \mathrm{~mL}$ YPM in syringes at an initial OD $_{600 \mathrm{~nm}}$ of 1 and 143 incubated under the same conditions as above. The plunger movement, as $\mathrm{CO}_{2}$ was accumulated, was 
144 recorded after every 2 hours for 20 hours. $\mathrm{CO}_{2}$ production yields were calculated by dividing the 145 amount accumulated at the end of fermentation by the biomass accumulated. In addition, $\mathrm{CO}_{2}$

146 production rate was calculated by determining the slope of the curve using the points at which the 147 accumulation of $\mathrm{CO}_{2}$ was the fastest. The same procedure was repeated with evolved lineages of $K$. 148 gamospora $\left(K_{g} \_1, K g \_2\right.$ and $\mathrm{Kg}_{-} 3$ ), both ancestral strains and a control conventional baker's yeast. 149 These experiments were done in triplicates and repeated three times.

\section{$150 \quad 2.6 \quad$ Investigation of leavening ability}

151 The evolved yeast isolates $\left(K g \_1, K g \_2, K g \_3, W s \_1, W s \_2\right.$, and $\left.W s_{-} 3\right)$, the ancestral strains and the control conventional baker's yeast were grown in $2 \mathrm{~mL}$ of $5 \%$ YPM in $5 \mathrm{~mL}$ tubes and incubated overnight at $26{ }^{\circ} \mathrm{C}$ at $200 \mathrm{rpm}$ on a shaker (Infors HT). The yeasts were then harvested by centrifugation and inoculated into fresh $20 \mathrm{~mL}$ YPM and put back on the shaker for another 24 hours to increase cell biomass. $2 \mathrm{~mL}$ of cells at an $\mathrm{OD}_{600 \mathrm{~nm}}$ of 10 was used to inoculate $10 \mathrm{~g}$ of flour dough in Falcon tubes. The Falcon tubes were left to ferment for 1 hour in a $30{ }^{\circ} \mathrm{C}$ incubator. The change in leavening was assessed as dough increased in volume. Respective volume increases after incubation were recorded by photographing. The experiment was done in triplicates and repeated three times.

\subsection{Investigation of stress tolerance}

161 The evolved yeast isolates $\left(K g \_1, K g \_2, K g \_3, W s \_1, W s \_2\right.$, and $\left.W s \_3\right)$, the ancestral strains and the 162 control conventional baker's yeast were grown overnight in $5 \%$ liquid YPM in $5 \mathrm{~mL}$ tubes at $26^{\circ} \mathrm{C}$ 163 at $200 \mathrm{rpm}$ on a shaker (Infors HT) as above. Cells were then harvested by centrifugation and then 164 washed twice with sterile deionized water. The cells were then adjusted to an initial OD $600 \mathrm{~nm}$ of 0.2.

165 The cells were then serially diluted ( 2 folds dilution ranges) in sterile phosphate buffer saline and pipetted into 96 well plates. A spot test stamp (replicator) was used to spot cells on solid YPD media plates supplemented with different stressors and incubated at $30{ }^{\circ} \mathrm{C}$ for 72 hours. For oxidative stress tolerance, yeasts were grown in YPD agar supplemented with hydrogen peroxide $\left(\mathrm{H}_{2} \mathrm{O}_{2}\right)$ at different concentrations ( $3 \mathrm{mM}, 4 \mathrm{mM}, 5 \mathrm{mM}, 6 \mathrm{mM}$ and $7 \mathrm{mM})$. For ethanol stress tolerance, yeasts were grown on YPD agar containing different concentrations of ethanol (5\%, $7 \%, 9 \%$ and $10 \%(\mathrm{v} / \mathrm{v}))$.

171 Similarly, for halotolerance, yeasts were grown in YPD agar supplemented with sodium chloride $172(\mathrm{NaCl})$ of different concentrations $(0.5 \mathrm{M}, 1 \mathrm{M}, 1.2 \mathrm{M}, 1.5 \mathrm{M}, 1.6 \mathrm{M}, 1.8 \mathrm{M}$ and $2 \mathrm{M})$. 173 Osmotolerance tests were carried out by growing cells on YPS agar at different concentrations of 
174 sucrose $(50 \%$ and $60 \%)$. For thermotolerance, yeasts cells were grown on YPM agar and incubated 175 at $30{ }^{\circ} \mathrm{C}, 37{ }^{\circ} \mathrm{C}, 40{ }^{\circ} \mathrm{C}, 42{ }^{\circ} \mathrm{C}$ and $44^{\circ} \mathrm{C}$. Growth of evolved clones was compared to the ancestral

176 strains as well as the control baker's yeasts and scored qualitatively. The experiments were done in

177 triplicates and repeated thrice. The best representative plates were scanned and recorded.

178

179

180

181

182

183

\subsection{Baking trials}

Leavened dough from the evolved yeast isolates $\left(K g \_1, K g \_2, K g \_3, W s \_1, W s \_2\right.$, and $\left.W s_{-} 3\right)$, the ancestral strains and the control conventional baker's yeast from Section 2.6 were used as starter cultures. $50 \mathrm{~g}$ of flour was added to each of the doughs and weighed before and after fermentation to determine the percentage change in weight. After fermentation, the dough was kneaded and moulded into greaseproof muffin moulds. The leavened and moulded dough was baked for 20 minutes at 250 ${ }^{\circ} \mathrm{C}$ and 15 minutes in a conventional oven until the bread developed a brownish crust. After baking, the bread was weighed and the percentage change in weight was recorded. The overall texture and pore sizes were photographed and recorded.

\subsection{Statistical Analyses}

To test whether independently evolved clones and the controls had significantly different fermentative capacity as a function of $\mathrm{CO}_{2}$ production rate, $\mathrm{CO}_{2}$ yields and cell-densities, one-way ANOVA was conducted. To test whether the same attributes of independently evolved lines significantly differed from each other, we implemented a post-hoc Tukey's HSD. The significance level was set at $\mathrm{p}<0.05, \mathrm{p}<0.01$ and $\mathrm{p}<0.001$. All the analyses were done using STATISTICA, version 13.2 (Statsoft Inc., Tulsa, Oklahoma).

\section{Results}

\subsection{Evolutionary engineering improved the fermentative capacity of 5 out of 6 independent lines}

Our previous work (Zhou et al., 2017) reported preliminary observation that potential alternative baking yeasts, Wickerhamomyces subpelliculosus and Kazachstania gamospora could be adaptively evolved in dough-like conditions. Due to a short incubation time, shorter passaging cycles and absence of characterisation experiments the results were insufficient to ascertain the evolvability and application of the strategy in improving alternative baker's yeasts with poor baking attributes. We were prompted to serially passage the yeasts for longer periods of incubation of $48 \mathrm{~h}$ (12 h longer) as 
well as increasing passaging cycles, in this case, until the Eppendorf tubes popped before the 204 predetermined incubation time. In addition, in this study we characterised the carbon dioxide 205 production rates and yield using maltose, the dough leavening ability and the improvement in stress tolerance before and after evolution and compared to conventional baker's yeast predominantly used in Southern Africa (S. cerevisiae, instant baker's yeast supplied by Anchor yeast, Co.). Here we report that at 60 passages the tubes started popping before the predetermined incubation time of $48 \mathrm{~h}$ suggestive of the improvement in fermentative rates. To confirm the suggestive improvements, we picked a single terminally evolved clone from each of the independently evolved lines and tested their fermentative capacity. Maltose was chosen for testing because it is the most abundant fermentable carbon source in wheat flour (Randez-Gil et al., 2013). Our results show that 5 out of 6 evolved clones accumulated on average 16.43 times more $\mathrm{CO}_{2}(49.28 \pm 3.36 \mathrm{~mL})$ at the end of fermentation (after $18 \mathrm{~h}$ ) as compared to their ancestral strains $(3.0 \pm 0.94 \mathrm{~mL})$ indicative of a tenfold improvement in the fermentative capacity using the most abundant carbon source in wheat flour (Figure 1A). In contrast, there was strangely no evident change in one of the six evolved clones from one of the parallel lines analysed $\left(\mathrm{Kg}_{-} 2\right)$. Analyses of ITS - ITS4 amplicons suggested that the clone was still $K$. gamospora, which probably lost the ability to ferment maltose efficiently. Overall, our approach was very effective as we observed 4.8 times more $\mathrm{CO}_{2}$ accumulated when compared to the control conventional baker's yeast $(10.3 \pm 1.15 \mathrm{~mL})$ within the first 18 hours of incubation. These results suggested that the ancestral strains and the control baker's yeasts are characterised by a longer lag phase during the utilisation of maltose as they later on managed to accumulate more carbon dioxide similar the amounts produced by the evolved clones (results not shown).

In addition to the ability to ferment maltose, we evaluated $\mathrm{CO}_{2}$ yield of the evolved clones as another important attribute required in leavening the dough. The results showed that there was a significant improvement in $\mathrm{CO}_{2}$ yield among 5 of the 6 evolved clones $\left(\mathrm{Kg}_{-} \mathrm{l}, \mathrm{Kg} g_{-} 3, W s_{-} 1, W s_{-} 2\right.$ and $\left.W s_{-} 3\right)$ when compared to the ancestral strains as well as the control baker's yeast (ANOVA, $p<0.001$ ) (Figure 1B). The evolved clones exhibited a $\mathrm{CO}_{2}$ yield that was eight times higher than that of the ancestral strains, which is a significant improvement. Again, $\mathrm{Kg}_{-} 2$ was an outlier. Our approach improved $\mathrm{CO}_{2}$ yield of 5 out of 6 evolved clones to two times more than that of the control baker's yeast, suggesting that the evolved clones would be a preferable alternative baker's yeasts for the baking industry. Interestingly, there was no significant difference in $\mathrm{CO}_{2}$ production, production rate and yield among these evolved clones (ANOVA, p < 0.001) (see Appendices, Table 4-Table 6) suggesting that the approach is independent of the background of the ancestral strain, which is a 
235 positive attribute to adopt the same strategy to other yeast species of interest. Another important 236 attribute of a model baker's yeast, the gassing power $\left(\mathrm{CO}_{2}\right.$ production rate), which reduces the time 237 taken to leaven dough, an important techno-economic factor (Giannone et al., 2010) was tested. We 238 observed a similar trend on the $\mathrm{CO}_{2}$ production rate, also known as the gassing power, an important 239 attribute determining the speed of dough leavening, among the 5 of the 6 evolved clones in

240 comparison to their ancestral strains and the control baker's yeast (Figure 1C). There was a highly 241 significant improvement in the gassing power of the 5 of the 6 evolved clones when compared to 242 ancestral strains as well as the commercial baker's' yeast (ANOVA, p < 0.001). In addition, there 243 was no statistical difference on gassing power attribute tested among the 5 out of 6 evolved clones 244 (Tukey’s HSD, p < 0.001) (see Supplementary Materials, Table 8 - Table 10).

\subsection{Evolved clones improved the leavening ability}

246 One of the important attributes of baker's yeast is the ability to leaven the dough, an important 247 quality index of a baker's yeast (Ahi et al., 2010). Therefore, the leavening abilities of the evolved 248 clones were evaluated by fermenting the dough and compared them to the dough leavened by the ancestral strains as well as the commercial baker's yeast. Results showed improved leavening ability 250 of the evolved clones $\mathrm{Kg}_{-} \mathrm{I}, \mathrm{Kg} \_2$ and $\mathrm{Kg} \_3$ in comparison to the commercial baker's yeast and their ancestral strain (Figure 2A). Surprisingly, $K g \_2$ showed the ability to leaven dough to a volume similar to $\mathrm{Kg} \_1$. This attribute not observed in the fermentation of maltose, suggesting that $\mathrm{Kg}_{-} 2$ fermented another carbon source found in flour other than maltose is worth investigating in future if at all this strain should be adopted for use in the baking industry. On the other hand, strains $W s_{-} l$, $255 W s \_2$ and $W s \_3$ leavened the dough to double the volume when compared to their ancestor as well as to the control baker's yeast (Figure 2B). These strains evolved from the W. subpelliculosus lineage and showed an even higher leavening ability as compared to the $K$. gamospora lineages $\left(K g \_1, K g \_2\right.$, $\left.258 \quad \mathrm{Kg} \_3\right)$.

\subsection{Evolved clones improved baking associated stress tolerance}

260 Other than fermentative capacity, the ability to withstand baking associated stresses from biomass 261 production to baking is another desirable attribute of a baker's yeast. Baker's yeast may encounter 262 stresses during baking and storage high osmotic pressure, high oxidative stress, high/low 263 temperatures, ethanol stress among others (Attfield, 1999). We firstly investigated the ability to 264 withstand ethanol, a product of dough fermentation, as an important attribute that allows higher 
265 efficiency of leavening ability. The evolved clones $W s_{-} 1, W s_{-} 2$ and $W s_{\_} 3$ were resistant to ethanol 266 up to $9 \%$, which is $2 \%$ higher than the amount tolerable to the conventional baker's yeast (Figure

267 3). This was a huge improvement in ethanol stress tolerance, as their ancestor did not grow on $5 \%$ 268 ethanol. On the other hand, the evolved lines from the $K$. gamospora background ( $K g_{-} \_$and $K g_{-}$3) 269 tolerated only up to $5 \%$ ethanol. An interesting observation was that $K g \_2$ in addition to its poor 270 utilisation of maltose as a carbon source it also did not tolerate ethanol when compared to its 271 ancestor.

272 Another important attribute of a baker's yeast is the ability to withstand high temperatures. 273 Downstream processing for the preparation of biomass involving drying, storage and rehydration 274 exerts thermal stress as well as oxidative stress to the yeasts (Randez-Gil et al., 2013). Our results 275 suggest that our approach significantly improved thermotolerance of $W s \_1, W s \_2$ and $W s \_3$ evolved 276 clones up to $40{ }^{\circ} \mathrm{C}$ as compared to their ancestor, which did not grow at $37^{\circ} \mathrm{C}$ (Figure 4). A similar 277 improvement in thermotolerance was observed with $\mathrm{Kg}_{-} \mathrm{l}$ and $\mathrm{Kg} g_{-}$, except that their ancestor could 278 tolerate $37^{\circ} \mathrm{C}$. $\mathrm{Kg} \_2$ once again was an outlier in the ability to withstand thermal stress. In addition, 279 the ability to withstand high osmotic stress (as high as $60 \%$ ), an attribute of interest for an ideal 280 baker's yeast was investigated. Our findings show that $K g \_2$ was more osmotolerant, a trait shared 281 by the parental strain (Anc Kg), than all its evolved counterparts (Figure 5). In addition, a similar 282 trend was also noted for halotolerance (Figure 6). Oxidative stress tolerance was also evaluated as a 283 critical attribute of a baker's yeast because yeasts are exposed to reactive oxygen species generated during dough fermentation. Our results suggest that our approach improved oxidative stress tolerance of all evolved lines (Figure 7).

\subsection{Evolved clones show improved baking attributes}

Baking trials were conducted to evaluate the relevant attributes and impression of the baked bread, which are crucial to fulfil consumer demands (Rouillé et al., 2010). Sliced portions of the bread baked with different yeasts were evaluated. Bread baked with evolved clones had higher loaf volume when compared to bread baked with their ancestral strain as well as the control conventional baker's yeast (ANOVA, p < 0.001) except bread baked with K.g_2. This is in agreement to the higher amounts of $\mathrm{CO}_{2}$ produced in maltose fermentation as the loaf volume is proportional to the amount of $\mathrm{CO}_{2}$ trapped in the gluten matrix of the dough (Struyf et al., 2017). Although the dough leavened using $K g \_2$ had risen to a height just like other evolved strains, the height of the bread was lower (4.9 $\mathrm{cm}$ ) than breads baked with the other evolved strains (Figure 8) (ANOVA, p $<0.001$ ). Our results 
suggest that the highest loaf was attainable using $W$. subpelicullosus derived strains (Ws_1,Ws_2 and $\left.W s \_3\right)(7.3 \pm 0.36 \mathrm{~cm})($ Figure 8$)$.

Another important factor in final quality of the bread is the pore sizes, which influences the texture of bread. Bread baked with evolved clones had much more bigger and uniform pore sizes when compared to bread baked with the ancestral strains as well as the control conventional baker's yeast (Figure 8). Our evolutionary engineering approach improved the baking traits as we observed an improvement in loaf volume and overall appearance of the bread baked with 5 out of 6 evolved clones when compared to both their ancestors and the baker's yeast. Bread baked with $\mathrm{Kg \_} 2$ was in agreement to poor attributes of other traits investigated above.

To further reveal the change in baking attributes, we investigated the change in weight of dough before and after fermentation as well as that of bread after baking. Change in weight is considered a desirable quality attribute for the best outcome of bread (Sanchez-Garcia et al., 2015). The results of change in weight of dough after fermentation and weight of dough after baking are shown in Figure 9. It should be noted that the best producer of $\mathrm{CO}_{2}$ should be the best to leaven dough and, hence, the best in producing the best quality of bread in terms of texture and size. Results show that leavening of the dough and bread baked using the evolved clones lost more weight after baking as compared to their ancestors and the control baker's yeast.

\section{Discussion}

315 Saccharomyces cerevisiae, the industrial workhorse of all times, remains the baker's yeast of choice 316 despite its limitations. The development of alternative baker's yeasts is attractive due to modern 317 baking associated with a huge change in preference for healthy lifestyles, improved foods, improved

318 resource utilisation efficiency, and diversity of food products in our generation's consumer driven 319 markets. This study examined the hypothesis that adaptive evolution of previously reported potential 320 baking yeasts strains in flour dough conditions improves their baking traits. Indeed, not only were the 321 strains evolved to efficiently ferment the most abundant carbon source in flour leading to an elevated 322 speed of leavening, but the strains also improved the ability to withstand baking-associated stress, as 323 well as improving the outcome of the bread. This work demonstrated a direct relationship between 324 evolutionary engineering and improvement in baking traits. To our knowledge, this work is the first 325 work to report on the benefits of evolutionary engineering of non-conventional yeasts for 
326 development of alternative baker's yeasts. Use of evolutionary engineering to improve specific 327 fermentation capabilities is well described (Kim et al., 2013), although not in the case of non328 conventional yeasts. This work is in agreement with reports that possible selection over several 329 decades, as a long term evolutionary engineering in fermentations, could be the main reason why the 330 conventional baker's yeast has the best baking attributes (Randez-Gil et al., 2013).

331 Our observations are consistent with our previous preliminary observations (Zhou et al., 2017) which 332 suggested that baking attributes can be improved by passaging potential baking yeasts using dough 333 conditions. Increased maltose fermentative capacity associated with higher leavening ability is 334 supported by our experimental results. Maltose is the most abundant sugar in wheat flour and 335 therefore a baker's yeast is desirable if it has an efficient maltose fermentation capacity (Struyf et al., 336 2017). Relatively short fermentation time and leavening ability within a short space of time is of 337 commercial interest. This work did not test the effects of our evolutionary engineering approach on 338 sweet dough, which contains sucrose and fructan. However, it should be noted that sucrose and 339 fructan are degraded within the first hour of fermentation, leaving only maltose to sustain 340 fermentation (Struyf et al., 2017). The ability to leaven dough relies on carbohydrate composition of 341 which efficient utilisation of these carbon sources is very important for an alternative baker's yeast 342 (Randez-Gil et al., 2003). Although the rate of fermentation of flour dough by S. cerevisiae is known 343 to be the best in the baking industry, the results presented here suggest that it is possible to develop 344 alternative baking strains with comparable or better baking traits than the conventional baker's yeast currently used in the baking industry.

346 Survival and performance under baking associated stresses is another important attribute of a baker's 347 yeast. Thermotolerance is one of the most relevant traits because yeasts are subjected to thermal 348 stress during preparation of biomass, transportation and during fermentation of dough (Panadero et $349 a l ., 2007)$. Here, we report that evolutionary engineering improved resistance to higher temperatures. 350 Another stress of importance is oxidative stress, which has a well-known effect on dough rheology 351 during bread making (Bonet et al., 2006). Since most yeast biological systems generate reactive 352 oxygen during growth (Sies, 2014), an alternative yeast should develop resistance to this stress. This 353 work suggests that the evolutionary approach exploited led to an improved resistance in oxidative 354 stress. In addition, yeasts fermentation of sugars in the flour dough produces ethanol which can 355 reduce rates of growth, fermentative capacity and cell viability (Nagodawithana et al., 1976). Ethanol 356 production also contributes to the increased rate of $\mathrm{H}_{2} \mathrm{O}_{2}$ diffusion into the cells and thereby 
increasing oxidative stress during dough fermentation (Banat et al., 1998). In this sense, it is worth mentioning that we observed an improved resistance to ethanol, extreme temperature and oxidative stresses. In this way, the alternative baker's yeast reported in this work showed the improvement of critical desirable phenotypes.

361 Overall, our study demonstrated the potential in developing alternative baking yeasts with improved 362 phenotypes. It also highlights how this approach leads to improved bread appearance as an important characteristic on which the acceptability of bread depends on. The volume and texture of the bread are the major attributes of desirable bread. Evolutionary engineering improved bread attributes as noted from the results. The loss of ability to ferment and leaven the dough exhibited by one strain in this work is not surprising because the loss of phenotype or trade-offs during evolution is well known (Zeyl, 2006, Kumar \& Gayen, 2011, Charlesworth et al., 2017, Van den Bergh et al., 2018). Further studies to reveal the observed phenotypes would be worth exploring.

369 Although there are multiple strategies to develop yeast strains towards specific industrial characteristics as extensively reviewed by Steensels et al. (2014), evolutionary engineering is one of the simplest, yet very powerful approach to develop non-recombinant strains for the baking industry (Deckers et al., 2020). The search for baking yeasts from natural biodiversity, genetic and other nongenetic improvements are associated with many drawbacks. Searching for baking yeasts from nature is attractive, but the traits of such yeasts meant for survival and reproduction in their natural environments are not directly transferrable to highly stressful man-made environments, such as baking, brewing or other industrial processes (Steensels et al., 2014). Before such strains are used, artificial strain development strategies are necessary (Nevoigt, 2008). Genetic improvements of food grade yeasts are controversial for consumer acceptance (Da Silva \& Srikrishnan, 2012). Non-genetic modification techniques, as evolutionary engineering, used to improve strains are therefore more attractive.

In conclusion, our work highlighted that evolutionary engineering is an attractive tool to improve the baking performance of non-conventional yeasts, which has been a major limitation for entrance into the market monopolised by Saccharomyces cerevisiae. However, further studies are required to reveal the molecular mechanisms behind the observed improvements. Other studies to investigate more attributes such as the ability to withstand other baking associated stresses, changes in aroma complexity after evolution as well as the ability to utilise other carbon sources are required to develop more efficient alternative baker's yeasts. 
389 Authors declare no conflict of interest.

3906 Author Contributions

391 T.S performed the experiments. N.Z conceived the experiments and partly designed the experiments.

392 T.S, T.B, A.G and Senior Author N.Z wrote the paper.

\section{$393 \quad 7 \quad$ Funding}

394 This work was funded by the Department of Research and Innovation, Undergraduate Funding, 395 Botswana University of Science and Technology (BIUST).

\section{$8 \quad$ References}

398 Ahi M, Hatamipour M \& Goodarzi AJDT (2010) Optimization of leavening activity of baker's yeast 399 during the spray-drying process. 28: 490-494.

400 Aldhous P (1990) Genetic engineering. Modified yeast fine for food. 344: 186-186.

401 Aslankoohi E, Herrera-Malaver B, Rezaei MN, Steensels J, Courtin CM \& Verstrepen KJJPO (2016)

402 Non-conventional yeast strains increase the aroma complexity of bread. 11: e0165126.

403 Attfield PVJNb (1997) Stress tolerance: the key to effective strains of industrial baker's yeast. 15:

404 1351-1357.

405 Banat I, Nigam P, Singh D, Marchant R, McHale AJwJoM \& Biotechnology (1998) Ethanol

406 production at elevated temperatures and alcohol concentrations: Part I-Yeasts in general. 14: 809407821.

408 Bonet A, Rosell C, Caballero PA, Gómez M, Pérez-Munuera I \& Lluch MA (2006) Glucose oxidase 409 effect on dough rheology and bread quality: A study from macroscopic to molecular level. Food

410 Chemistry 99: 408-415.

411 Botstein D, Chervitz SA \& Cherry MJS (1997) Yeast as a model organism. 277: 1259-1260.

412 Charlesworth D, Barton NH \& Charlesworth BJPotRSBBS (2017) The sources of adaptive variation. 413 284: 20162864.

414 Da Silva NA \& Srikrishnan SJFyr (2012) Introduction and expression of genes for metabolic 415 engineering applications in Saccharomyces cerevisiae. 12: 197-214.

416 Deckers M, Deforce D, Fraiture M-A \& Roosens NHJF (2020) Genetically Modified Micro417 Organisms for Industrial Food Enzyme Production: An Overview. 9: 326.

418 Fay JC \& Benavides JAJPG (2005) Evidence for domesticated and wild populations of 419 Saccharomyces cerevisiae. 1: e5. 
Gamero A, Quintilla R, Groenewald M, Alkema W, Boekhout T \& Hazelwood LJFm (2016) Highthroughput screening of a large collection of non-conventional yeasts reveals their potential for aroma formation in food fermentation. 60: 147-159.

423 Gamero Lluna A \& de Jong CJNFM (2013) Novel yeasts, novel flavours. 16: 26-28.

424 Giannone V, Longo C, Damigella A, Raspagliesi D, Spina A, Palumbo MJJoim \& biotechnology 425 (2010) Technological properties of baker's' yeasts in durum wheat semolina dough. 37: 371-379.

426 Gibson B, Geertman J, Hittinger C, Krogerus K, Libkind D, Louis EJ, Magalhães F \& Sampaio JJFYR (2017) New yeasts - new brews: modern approaches to brewing yeast design and development. 17. C \& Johnston MJS (1996) Life with 6000 genes. 274: 546-567. strategy evolved as a multi-step process that predates the whole genome duplication. 8: e68734. Harvey JE, Choi N, Krywonos A, Peterson GL \& Bruner MEJOE (2010) Image degradation due to scattering effects in two-mirror telescopes. 49: 063202.

Hernández-López J \& Vargas-Albores FJAR (2003) A microplate technique to quantify nutrients (NO2-, NO3-, NH4+ and PO43-) in seawater. 34: 1201-1204.

Kariluoto S, Vahteristo L, Salovaara H, Katina K, Liukkonen KH \& Piironen VJCC (2004) Effect of baking method and fermentation on folate content of rye and wheat breads. 81: 134-139.

Kim SR, Park Y-C, Jin Y-S \& Seo J-HJBa (2013) Strain engineering of Saccharomyces cerevisiae for enhanced xylose metabolism. 31: 851-861.

Kumar M \& Gayen KJAE (2011) Developments in biobutanol production: new insights. 88: 19992012. Koufopanou VJN (2009) Population genomics of domestic and wild yeasts. 458: 337-341.

Nagodawithana TW, Steinkraus KHJA \& Microbiology E (1976) Influence of the rate of ethanol production and accumulation on the viability of Saccharomyces cerevisiae in" rapid fermentation". 31: $158-162$.

Nevoigt E (2008) Progress in Metabolic Engineering of Saccharomyces cerevisiae. 72: 379-412. Ostergaard S, Olsson L, Nielsen JJM \& reviews mb (2000) Metabolic engineering of Saccharomyces cerevisiae. 64: 34-50. fermentative capacity of baker's yeast. 73: 4824-4831. (2015) Looking beyond Saccharomyces: the potential of non-conventional yeast species for desirable traits in bioethanol fermentation. 15. 
461 Randez-Gil F, Aguilera J, Codón A, Rincón AM, Estruch F \& Prieto JA (2003) Baker's yeast:

462 challenges and future prospects. Functional genetics of industrial yeasts, 57-97. Springer.

463 Ravasio D, Carlin S, Boekhout T, Groenewald M, Vrhovsek U, Walther A \& Wendland JJF (2018)

464 Adding flavor to beverages with non-conventional yeasts. 4: 15.

465 Rouillé J, Chiron H, Colonna P, Della Valle G \& Lourdin DJJoCS (2010) Dough/crumb transition

466 during French bread baking. 52: 161-169.

467 Sanchez-Garcia M, Álvaro F, Peremarti A, Martín-Sánchez JA \& Royo CJEJoA (2015) Changes in

468 bread-making quality attributes of bread wheat varieties cultivated in Spain during the 20th century.

469 63: 79-88.

470 Sies HJJoBC (2014) Role of metabolic H2O2 generation redox signaling and oxidative stress. 289:

$471 \quad 8735-8741$.

472 Steensels J, Snoek T, Meersman E, Nicolino MP, Voordeckers K \& Verstrepen KJJFmr (2014)

473 Improving industrial yeast strains: exploiting natural and artificial diversity. 38: 947-995.

474 Struyf N, Van der Maelen E, Hemdane S, Verspreet J, Verstrepen KJ, Courtin CMJCRiFS \& Safety 475 F (2017) Bread dough and baker's yeast: An uplifting synergy. 16: 850-867.

476 Takagi H (2017) Construction of baker's yeast strains with enhanced tolerance to baking-associated 477 stresses. Biotechnology of yeasts and filamentous fungi,. 63-85. Springer.

478 Van den Bergh B, Swings T, Fauvart M, Michiels JJM \& Reviews MB (2018) Experimental design, 479 population dynamics, and diversity in microbial experimental evolution. 82.

480 Zeyl CJFyr (2006) Experimental evolution with yeast. 6: 685-691.

481 Zhou N, Schifferdecker AJ, Gamero A, Compagno C, Boekhout T, Piškur J \& Knecht WJIjofm 482 (2017) Kazachstania gamospora and Wickerhamomyces subpelliculosus: Two alternative baker's 483 yeasts in the modern bakery. 250: $45-58$. 


\section{$9 \quad$ List of Figure Legends}

487 Figure 1. Fermentative capability of evolved clones in comparison to their ancestors.

488 A. $\mathrm{CO}_{2}$ production profile $\mathbf{B} . \mathrm{CO}_{2}$ yield C. $\mathrm{CO}_{2}$ production rate. Anc $\mathrm{Kg}$ is an ancestor for $K g_{-} \_1$ $489 \quad K g \_2$ and $K g \_3$ strains, Anc Ws is an ancestor for $W s_{-} 1, W s_{-} 2, W s_{-} 3$ strains and the baker's yeast as 490 a control. The evolved clones show elevated fermentative capacity within $18 \mathrm{~h}$ of fermentation 491 (profile of fermentation after $18 \mathrm{~h}$ was excluded for brevity). This experiment was performed in 492 triplicates and repeated twice. Error bars represent the standard deviation from the mean (see 493 Supplementary Materials, Table 1-Table 3).

494 Figure 2. Qualitative leavening abilities of strains after being adaptively evolved in flour dough.

495 A. Left to right: control with no yeast (NC), control baker's yeast (control), ancestral K. gamospora 496 and evolved clones $\left(K g \_1, K g \_2, K g \_3\right)$ B. Left to right: control with no yeast (NC), control baker's 497 yeast, ancestral W. subpelliculosus and evolved strains. The evolved clones showed improved 498 leavening ability. Images were taken after an hour of incubation at room temperature.

Figure 3. Ethanol stress tolerance of the evolved clones.

500 Parental strains Anc $K g$ and Anc $W s$, evolved clones and commercial baker's yeast were spotted

$501\left(\mathrm{OD}_{600 \mathrm{~nm}}\right.$ 0.2, 0.1 and 0.05) for growth on YPM media supplemented with different ethanol 502 concentrations $(5 \%, 7 \%, 9 \%$, and $10 \%)$. The evolved clones show improved ethanol tolerance as 503 compared to the ancestral strains and conventional baker's yeast.

504 Figure 4. Thermotolerance of the evolved clones.

505 Parental strains Anc Kg and Anc Ws, evolved clones and commercial baker's yeast were spotted $506\left(\mathrm{OD}_{600 \mathrm{~nm}} 0.2,0.1\right.$ and 0.05$)$ for growth on YPM incubated at different temperatures $\left(30^{\circ} \mathrm{C}, 37^{\circ} \mathrm{C}, 40\right.$ $507{ }^{\circ} \mathrm{C}, 42^{\circ} \mathrm{C}$ and $44^{\circ} \mathrm{C}$ ). 5 out of 6 evolved clones show improved thermotolerance in contrast with the 508 ancestral strains and the conventional baker's yeast. $K g \_2$ lost its ability to withstand thermal stress 509 as compared to its ancestor.

510 Figure 5. Osmotolerance of the evolved clones. 
511 Parental strains Anc Kg and Anc Ws, evolved clones and commercial baker's yeast were spotted

$512\left(\mathrm{OD}_{600 \mathrm{~nm}}\right.$ 0.2, 0.1 and 0.05$)$ for growth on YPS media supplemented with different concentrations of

513 sucrose $(50 \%$ and $60 \%)$. Ws_1,Ws_2, Ws_3 and $K g \_2$ evolved clones retained a similar

514 osmotolerance capability compared to parental strains. $K g_{-} 1$ and $K g_{-} 3$ lost the osmotolerance trait.

\section{Figure 6. Halotolerance of the evolved clones.}

516 Parental strains Anc $\mathrm{Kg}$ and Anc Ws, evolved clones and commercial baker's yeast were spotted

$517\left(\mathrm{OD}_{600 \mathrm{~nm}}\right.$ 0.2, 0.1 and 0.05$)$ for growth on YPM media supplemented with different $\mathrm{NaCl}$

518 concentrations $(0.5 \mathrm{M}, 1 \mathrm{M}, 1.2 \mathrm{M}, 1.5 \mathrm{M}, 1.6 \mathrm{M}, 1.8 \mathrm{M}$ and $2 \mathrm{M})$. $K g_{-} 1$ and $K g_{-} 3$ lost the

519 halotolerance ability whereas $K g \_2$ retained the attribute. $W s \_1, W s \_2$ and $W s \_3$ strains maintained

520 their poor halotolerance as their ancestral strain (Anc Ws).

521 Figure 7. Oxidative stress tolerance of the evolved clones.

522 Parental strains Anc Kg and Anc Ws, evolved clones and commercial baker's yeast were spotted

$523\left(\mathrm{OD}_{600 \mathrm{~nm}}\right.$ 0.2, 0.1 and 0.05) for growth on YPM media supplemented with different $\mathrm{H}_{2} \mathrm{O}_{2}$

524 concentrations ( $3 \mathrm{mM}, 4 \mathrm{mM}, 5 \mathrm{mM}, 6 \mathrm{mM}$ and $7 \mathrm{mM})$. All the evolved clones showed improved

525 oxidative stress tolerance as compared to the ancestral strains and the conventional baker's yeast.

526 Figure 8. Images of cross section of breads baked with different yeast clones.

527 Left to right: Unleavened bread NC (without yeast), control baker's yeast (control), ancestral strains 528 (Anc $K g$ and Anc $W s$ ) followed by their respective evolved strains. The height of each loaf was 529 recorded and used for comparing the loaf volumes after baking with a respective yeast strain.

530 Figure 9. Percentage change in weight of the dough after fermentation and after baking.

531 Evolved clones show more weight change as compared to their ancestral strains and the control 532 baker's yeast. (See also Supplementary Materials, Table 4). 
A

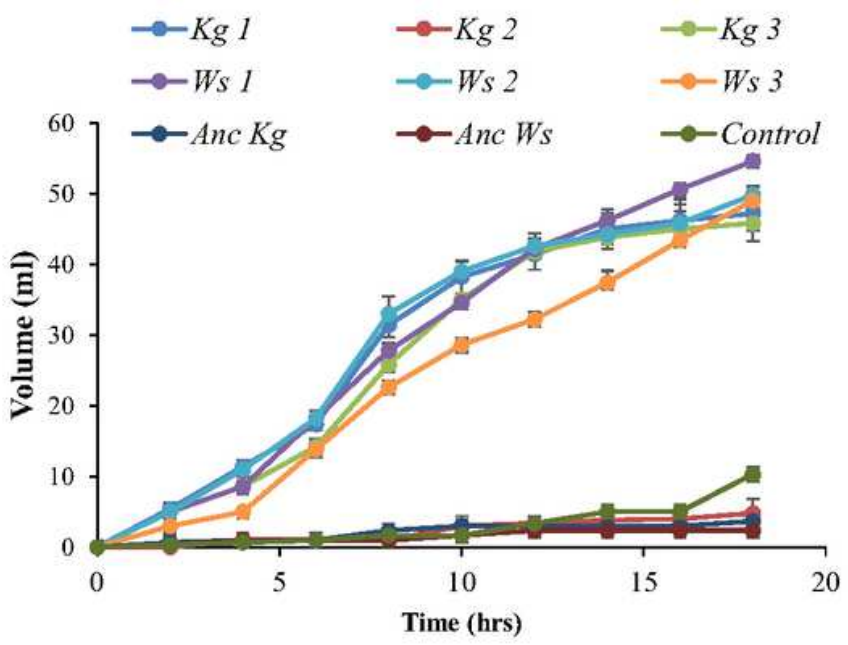

B

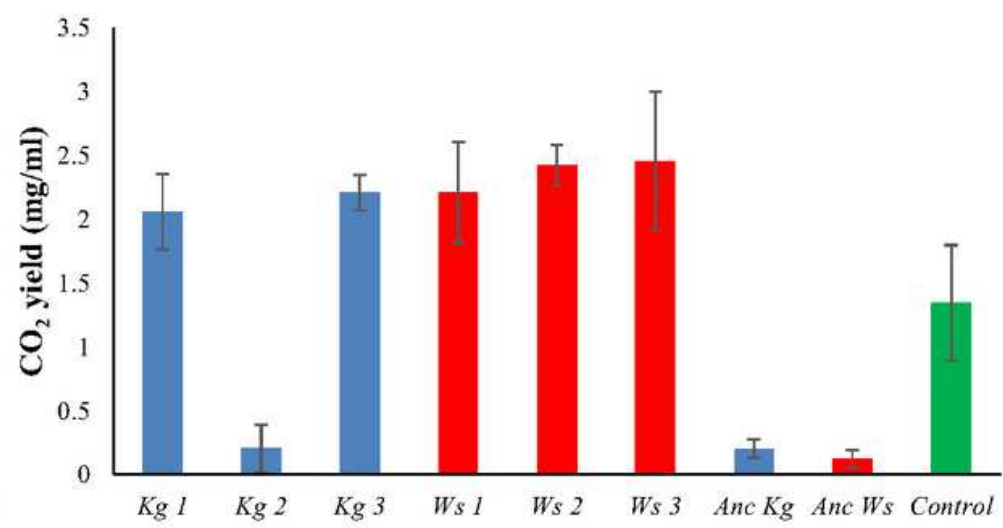

C

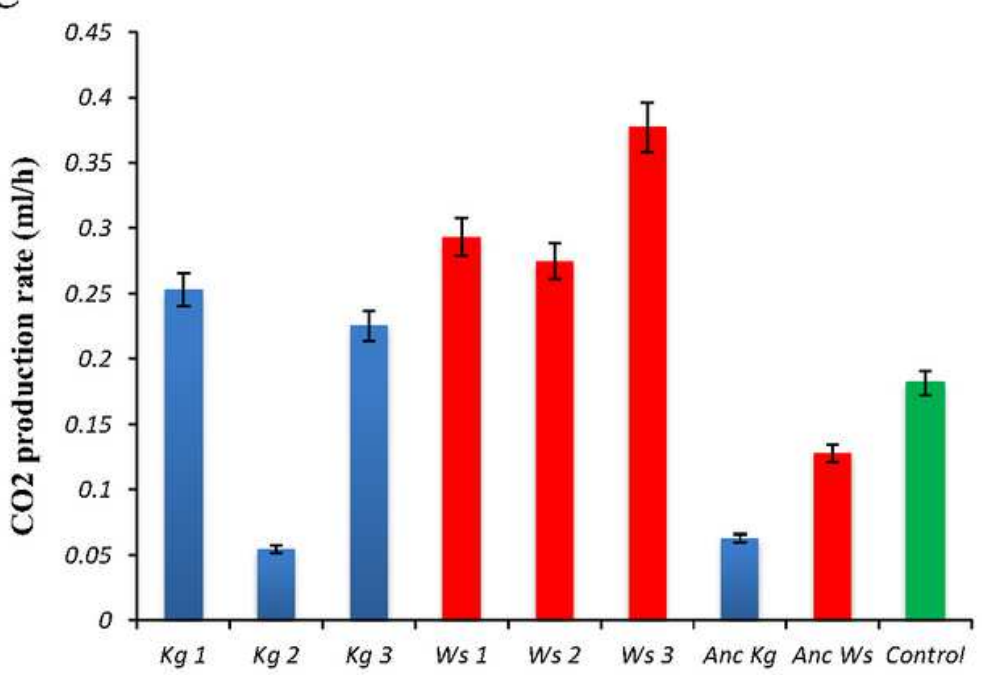

Figure 1

Fermentative capability of evolved clones in comparison to their ancestors. A. CO2 production profile B. $\mathrm{CO} 2$ yield C. $\mathrm{CO} 2$ production rate. Anc $\mathrm{Kg}$ is an ancestor for Kg_1 Kg_2 and Kg_3 strains, Anc Ws is an ancestor for Ws_1, Ws_2, Ws_3 strains and the baker's yeast as a control. The evolved clones show elevated fermentative capacity within $18 \mathrm{~h}$ of fermentation (profile of fermentation after $18 \mathrm{~h}$ was excluded for brevity). This experiment was performed in triplicates and repeated twice. Error bars represent the standard deviation from the mean (see Supplementary Materials, Table 1-Table 3). 


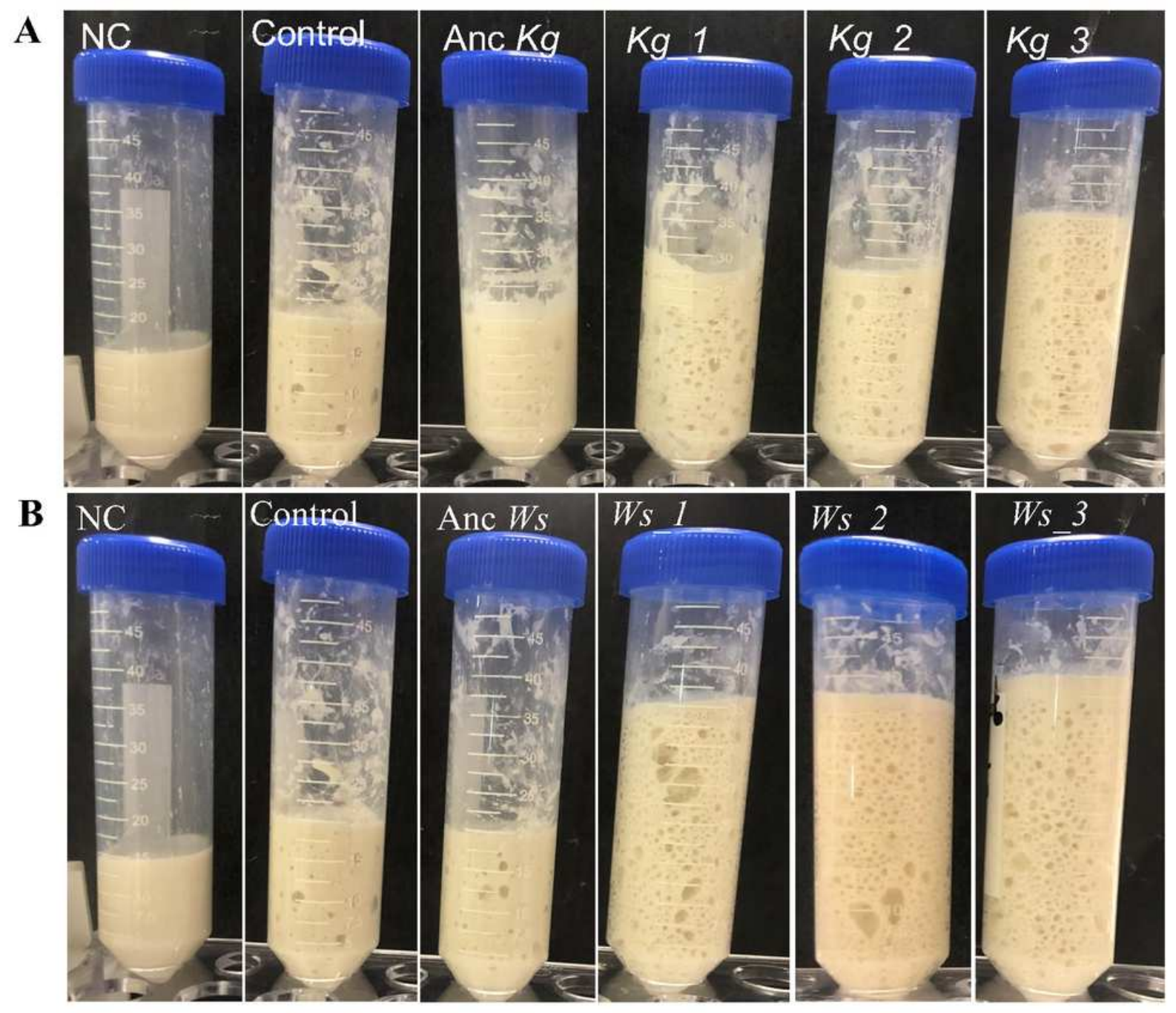

Figure 2

Qualitative leavening abilities of strains after being adaptively evolved in flour dough. A. Left to right: control with no yeast (NC), control baker's yeast (control), ancestral K. gamospora and evolved clones (Kg_1, Kg_2, Kg_3) B. Left to right: control with no yeast (NC), control baker's yeast, ancestral W. subpelliculosus and evolved strains. The evolved clones showed improved leavening ability. Images were taken after an hour of incubation at room temperature. 


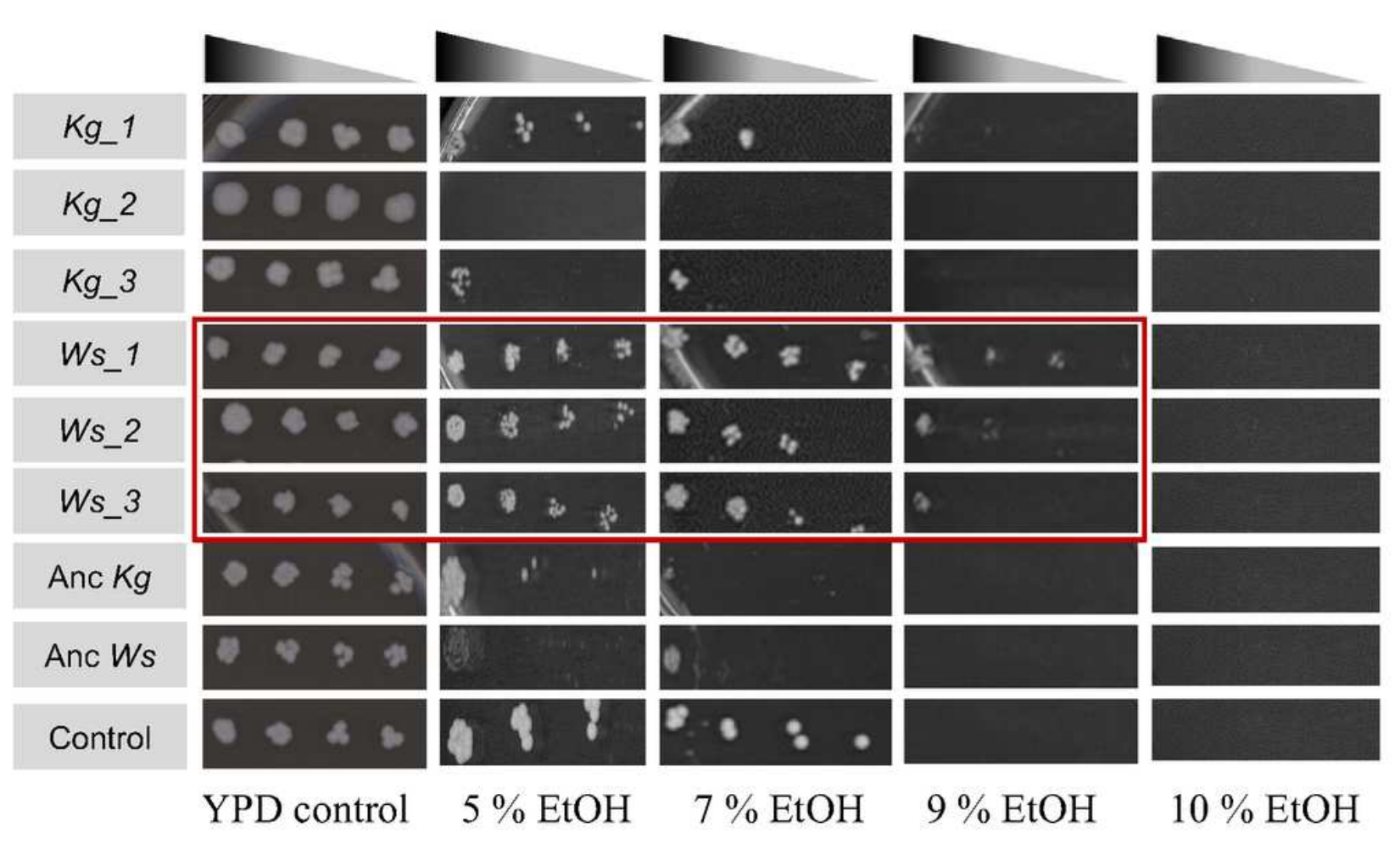

\section{Figure 3}

Ethanol stress tolerance of the evolved clones. Parental strains Anc $\mathrm{Kg}$ and Anc Ws, evolved clones and commercial baker's yeast were spotted (OD600nm 0.2, 0.1 and 0.05) for growth on YPM media supplemented with different ethanol concentrations (5\%, 7\%, $9 \%$, and $10 \%)$. The evolved clones show improved ethanol tolerance as compared to the ancestral strains and conventional baker's yeast. 


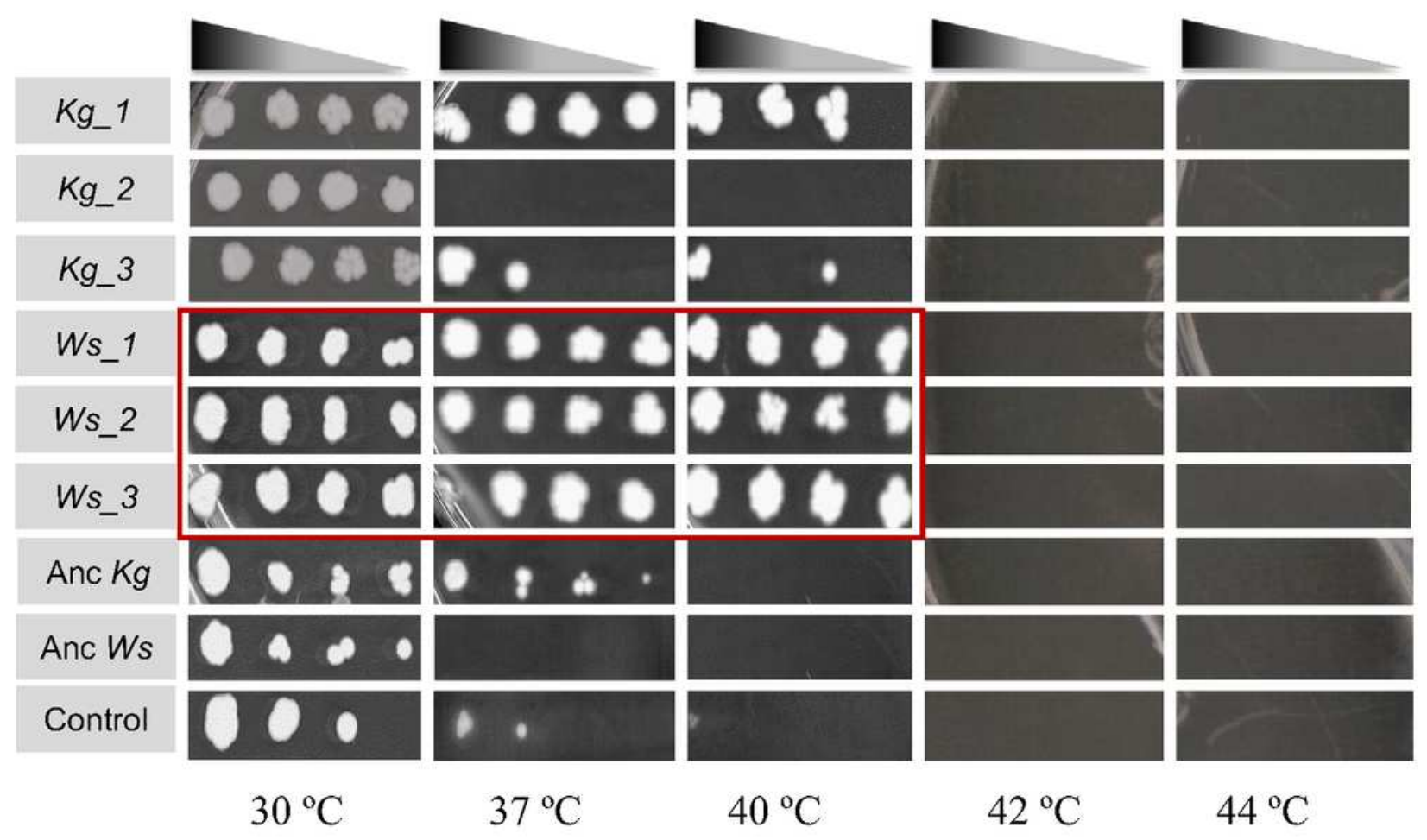

Figure 4

Thermotolerance of the evolved clones. Parental strains Anc Kg and Anc Ws, evolved clones and commercial baker's yeast were spotted (OD600 nm 0.2, 0.1 and 0.05) for growth on YPM incubated at different temperatures $\left(30^{\circ} \mathrm{C}, 37^{\circ} \mathrm{C}, 40506^{\circ} \mathrm{C}, 42^{\circ} \mathrm{C}\right.$ and $\left.44^{\circ} \mathrm{C}\right) .5$ out of 6 evolved clones show improved thermotolerance in contrast with the ancestral strains and the conventional baker's yeast. Kg_2 lost its ability to withstand thermal stress as compared to its ancestor. 


\section{Osmotolerance}

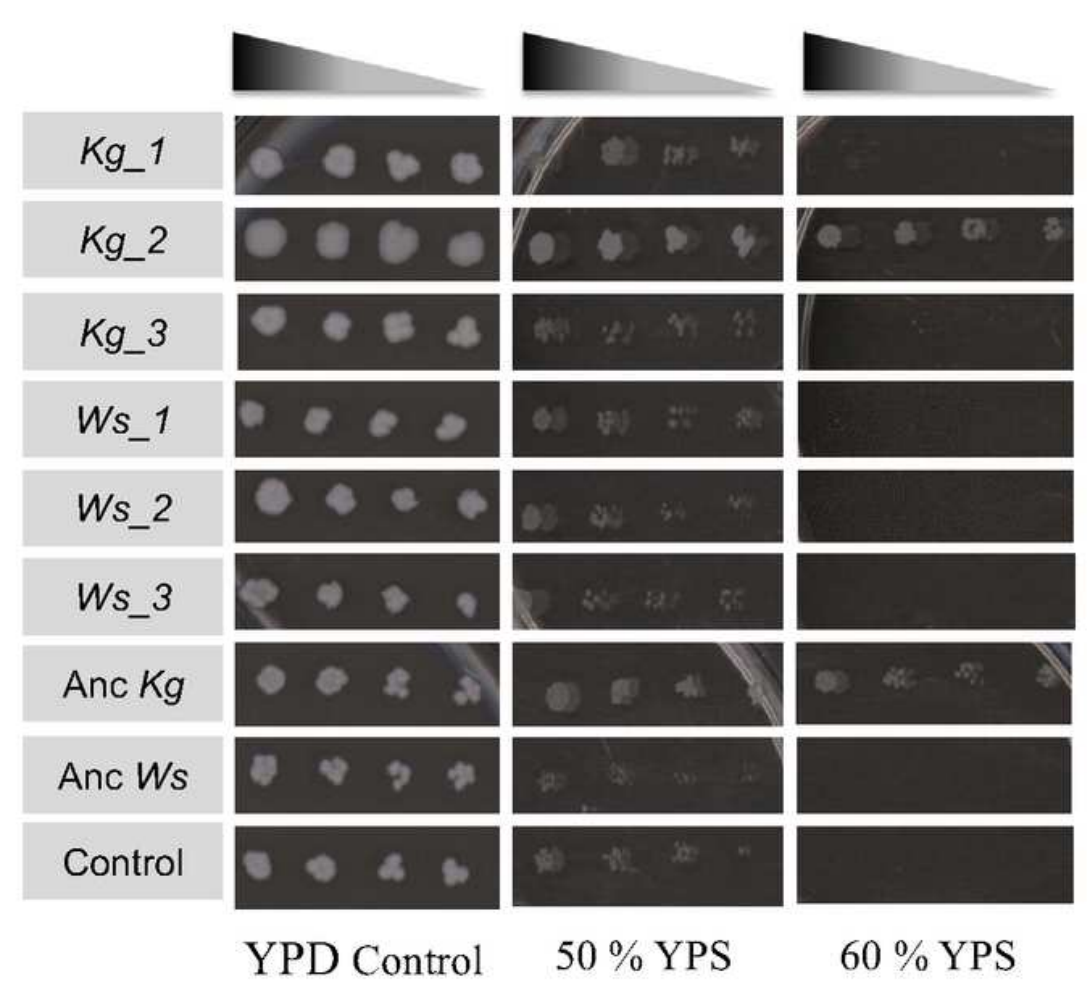

Figure 5

Osmotolerance of the evolved clones. 18 Parental strains Anc Kg and Anc Ws, evolved clones and commercial baker's yeast were spotted (OD600nm 0.2, 0.1 and 0.05) for growth on YPS media supplemented with different concentrations of sucrose (50 \% and $60 \%)$. Ws_1, Ws_2, Ws_3 and Kg_2 evolved clones retained a similar osmotolerance capability compared to parental strains. Kg_1 and Kg_3 lost the osmotolerance trait. 


\section{Halotolerance}

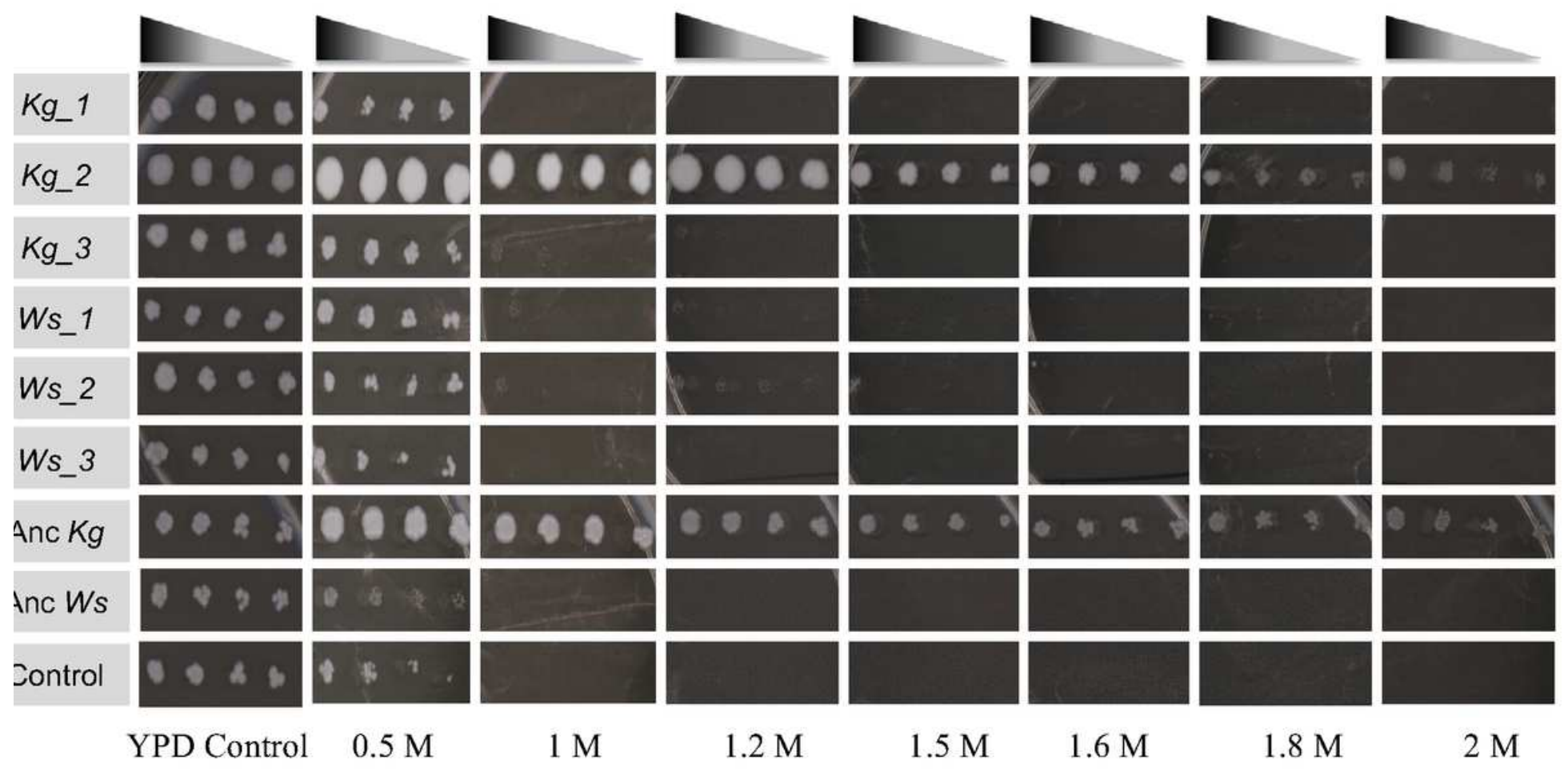

Figure 6

Halotolerance of the evolved clones. Parental strains Anc Kg and Anc Ws, evolved clones and commercial baker's yeast were spotted (OD600nm 0.2, 0.1 and 0.05) for growth on YPM media supplemented with different $\mathrm{NaCl}$ concentrations $(0.5 \mathrm{M}, 1 \mathrm{M}, 1.2 \mathrm{M}, 1.5 \mathrm{M}, 1.6 \mathrm{M}, 1.8 \mathrm{M}$ and $2 \mathrm{M})$. Kg_1 and Kg_3 lost the halotolerance ability whereas $\mathrm{Kg} \_2$ retained the attribute. Ws_1, Ws_2 and Ws_3 strains maintained their poor halotolerance as their ancestral strain (Anc Ws). 


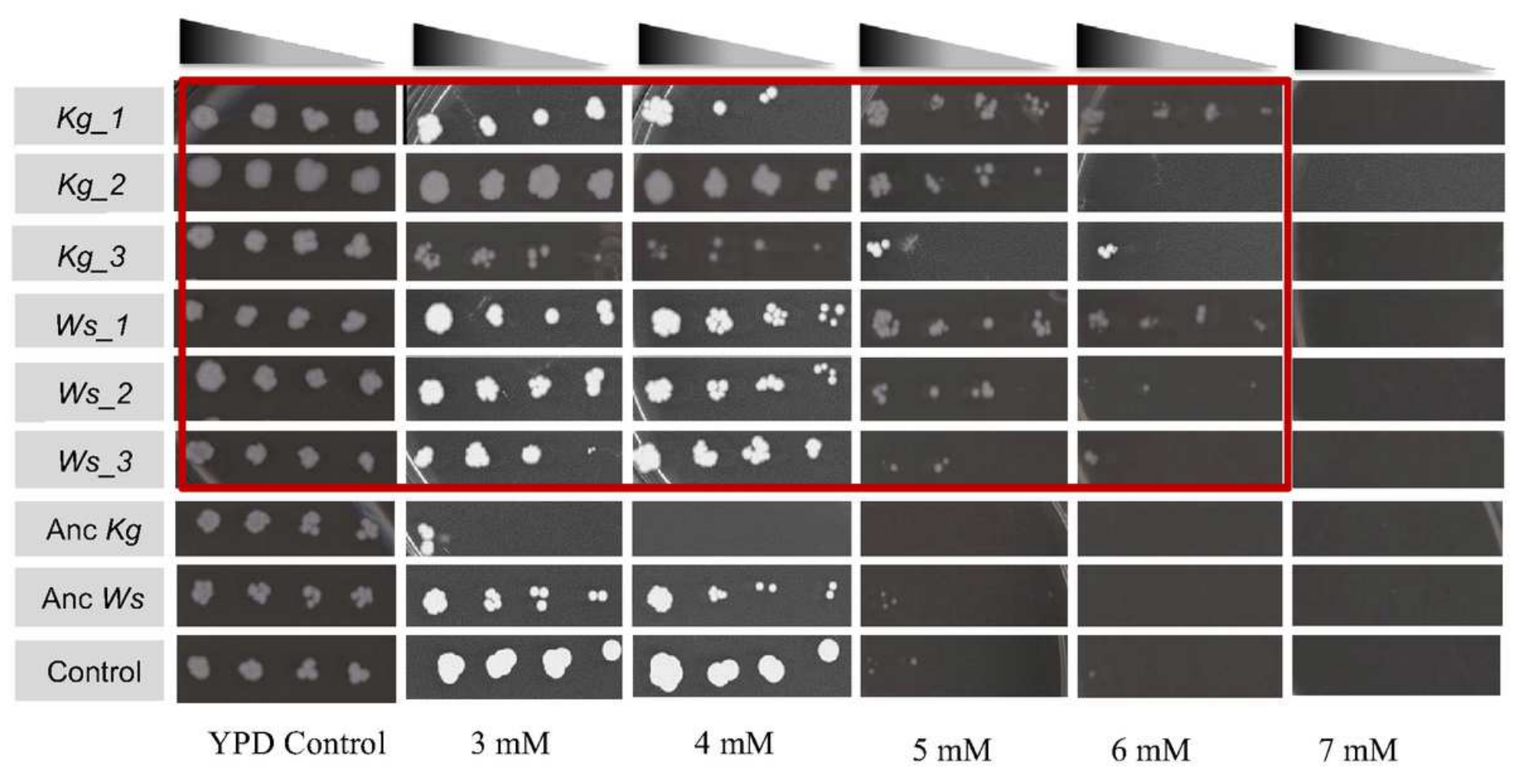

Figure 7

Oxidative stress tolerance of the evolved clones. Parental strains Anc $\mathrm{Kg}$ and Anc Ws, evolved clones and commercial baker's yeast were spotted (OD600nm 0.2, 0.1 and 0.05) for growth on YPM media supplemented with different $\mathrm{H} 2 \mathrm{O} 2$ concentrations ( $3 \mathrm{mM}, 4 \mathrm{mM}, 5 \mathrm{mM}, 6 \mathrm{mM}$ and $7 \mathrm{mM}$ ). All the evolved clones showed improved oxidative stress tolerance as compared to the ancestral strains and the conventional baker's yeast. 


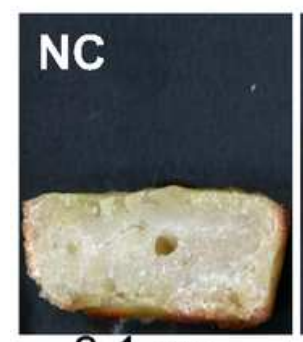

$3.1 \mathrm{~cm}$

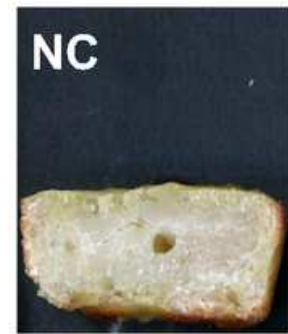

$3.1 \mathrm{~cm}$

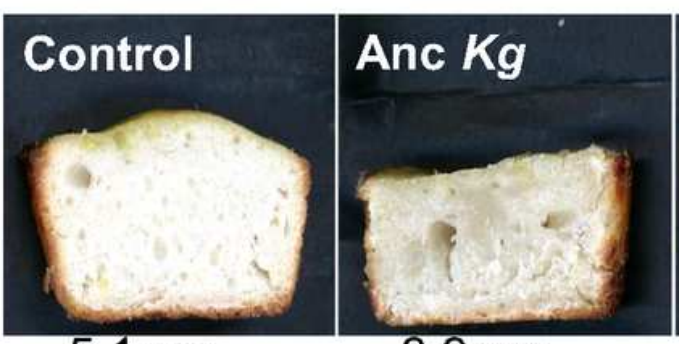

$5.1 \mathrm{~cm}$

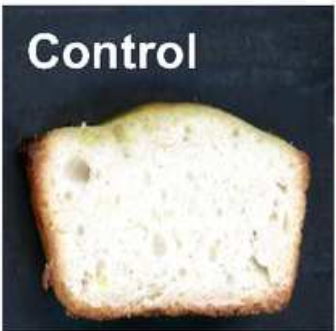

$5.1 \mathrm{~cm}$

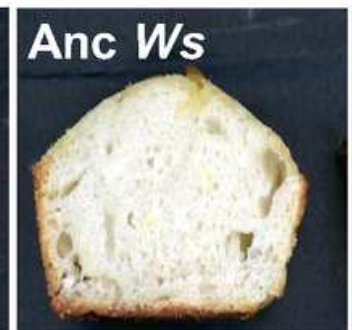

$6.1 \mathrm{~cm}$

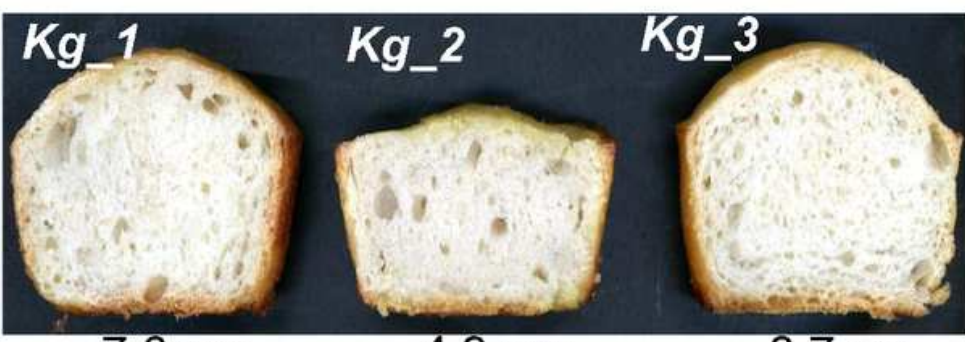

$7.0 \mathrm{~cm}$

$4.9 \mathrm{~cm}$

$6.7 \mathrm{~cm}$

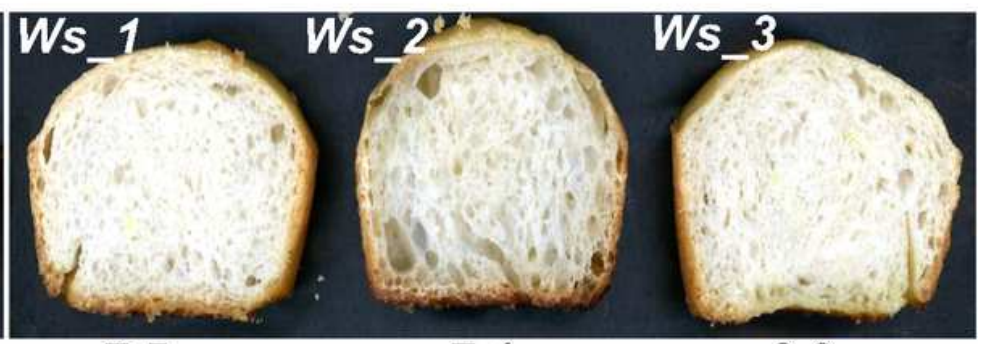

$7.4 \mathrm{~cm}$

$6.9 \mathrm{~cm}$

\section{Figure 8}

Images of cross section of breads baked with different yeast clones. Left to right: Unleavened bread NC (without yeast), control baker's yeast (control), ancestral strains (Anc Kg and Anc Ws) followed by their respective evolved strains. The height of each loaf was ecorded and used for comparing the loaf volumes after baking with a respective yeast strain. 


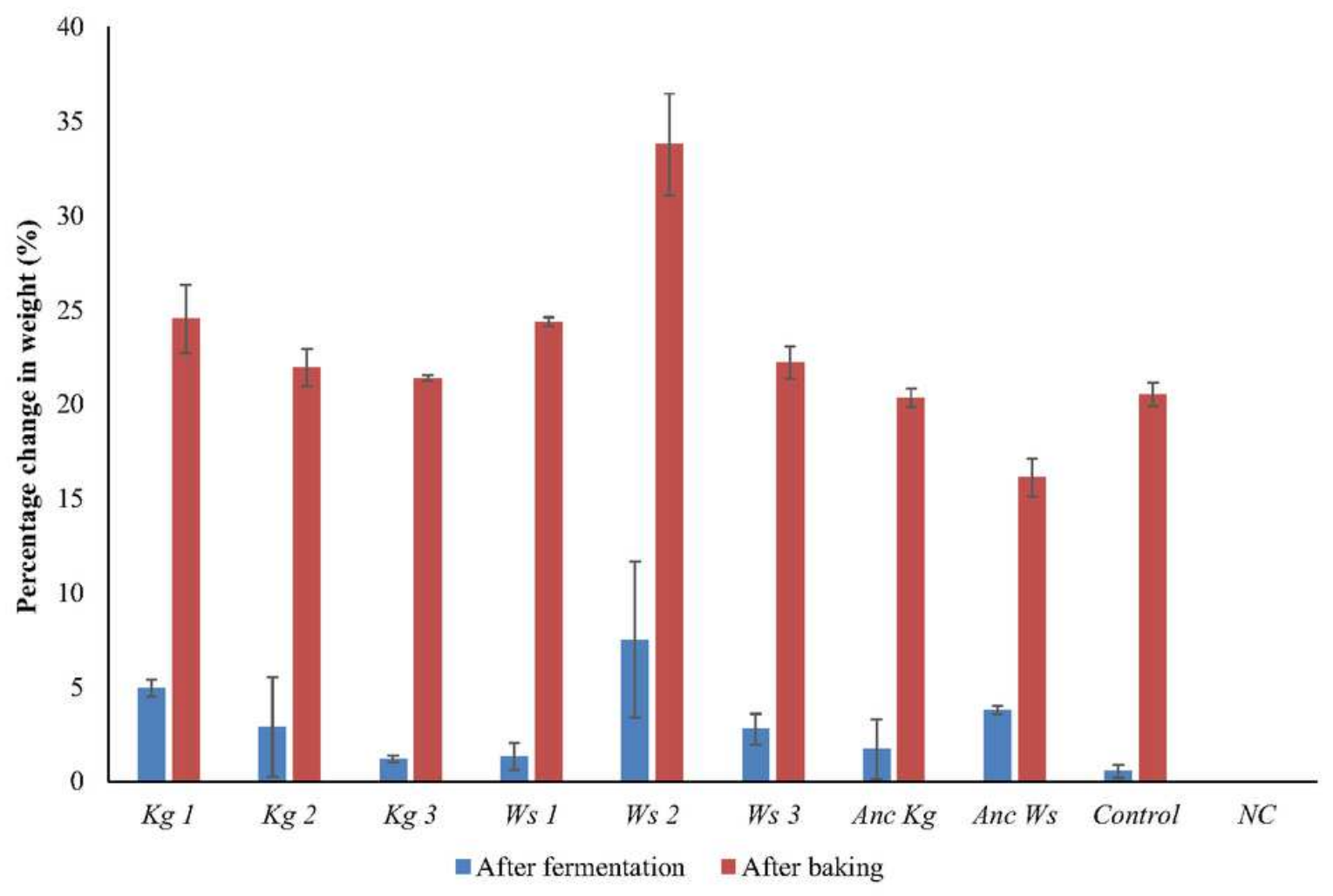

Figure 9

Percentage change in weight of the dough after fermentation and after baking. Evolved clones show more weight change as compared to their ancestral strains and the control 531 baker's yeast. (See also Supplementary Materials, Table 4).

\section{Supplementary Files}

This is a list of supplementary files associated with this preprint. Click to download.

- Supplementarymaterials.docx 